\title{
Challenges in String and Supersymmetric Cosmology
}

\author{
Ignatios Antoniadis, ${ }^{1,2}$ and Auttakit Chatrabhuti ${ }^{3}$ \\ ${ }^{1}$ Laboratoire de Physique Théorique et Hautes Energies - LPTHE, Sorbonne Université, CNRS, 4 Place Jussieu, 75005 Paris, France \\ ${ }^{2}$ Albert Einstein Center for Fundamental Physics, Institute for Theoretical Physics, University of Bern, Sidlerstrasse 5, CH-3012 Bern, \\ Switzerland \\ ${ }^{3}$ Department of Physics, Faculty of Science, Chulalongkorn University, Phayathai Road, Pathumwan, Bangkok 10330 , Thailand
}

\begin{abstract}
We discuss the possibility that inflation is driven by supersymmetry breaking with the superpartner of the goldstino (sgoldstino) playing the role of the inflaton. Imposing an R-symmetry allows to satisfy easily the slow-roll conditions, avoiding the so-called $\eta$-problem, and leads to an interesting class of small field inflation models, characterised by an inflationary plateau around the maximum of the scalar potential near the origin, where R-symmetry is restored with the inflaton rolling down to a minimum describing the present phase of the Universe. Inflation can be driven by either an F- or a D-term, while the minimum has a positive tuneable vacuum energy. The models agree with cosmological observations and in the simplest case predict a rather small tensor-to-scalar ratio of primordial perturbations.
\end{abstract}

Keywords: supersymmetry, supergravity, cosmology, inflation

DOI: 10.31526/ ACP.NDM-2020.6

\section{INTRODUCTION}

A fundamental theory of Nature, such as string theory, should be able to describe at the same time particle physics and cosmology, which are phenomena that involve very different scales from the microscopic four-dimensional (4d) quantum gravity length of $10^{-33} \mathrm{~cm}$ to large macroscopic distances of the size of the observable Universe $\sim 10^{28} \mathrm{~cm}$, spanned a region of about 60 orders of magnitude. In particular, besides the $4 \mathrm{~d}$ Planck mass, there are three very different scales with very different physics corresponding to the electroweak scale, dark energy and inflation. An interesting possibility is that these scales are related by the dynamics of an underlying fundamental theory, such as string theory. A first step towards this goal is to study possible connections between the electroweak scale of the Standard Model or its possible extension (such as the supersymmetry breaking scale) with that of inflation. An additional constraint would be to impose at the electroweak vacuum the presence of a tiny tuneable cosmological constant in order to accommodate the observed dark energy, without necessarily trying to explain it. Indeed, despite the absence of evidence of low-energy supersymmetry at colliders, it is likely theoretically that supersymmetry plays a role at some more fundamental level. On the other hand, inflation is an attractive paradigm for cosmology but the associated models provide a phenomenological description leaving several unanswered questions, such as the origin of the inflaton field, its fundamental or composite nature and its connection to the rest of particle physics.

Inflationary models in supergravity ${ }^{1}$ suffer in general from several problems, such as fine-tuning to satisfy the slow-roll conditions, large field initial conditions that break the validity of the effective field theory, and stabilisation of the (pseudo) scalar companion of the inflaton arising from the fact that bosonic components of superfields are always even. The simplest argument to see the fine tuning of the potential is that a canonically normalised kinetic term of a complex scalar field $X$ corresponds to a quadratic Kähler potential $K=X \bar{X}$ that brings one unit contribution to the slow-roll parameter $\eta=V^{\prime \prime} / V$, arising from the $e^{K}$ proportionality factor in the expression of the scalar potential $V$. This problem can be avoided in models with no-scale structure where cancellations arise naturally due to non-canonical kinetic terms leading to potentials with flat directions (at the classical level). However, such models require often trans-Planckian initial conditions that invalidate the effective supergravity description during inflation. A concrete example where all these problems appear is the Starobinsky model of inflation [2], despite its phenomenological success.

All three problems above are solved when the inflaton is identified with the scalar component of the goldstino superfield ${ }^{2}$, in the presence of a gauged R-symmetry [4]. Indeed, the superpotential is in that case linear and the big contribution to $\eta$ described above cancels exactly. Since inflation arises at a plateau around the maximum of the scalar potential (hill-top) no large field initial conditions are needed, while the pseudo-scalar companion of the inflaton is absorbed into the R-gauge field that becomes massive, leading the inflaton as a single scalar field present in the low-energy spectrum. This model provides therefore a minimal realisation of natural small-field inflation in supergravity, compatible with present observations, as we show below. Moreover, it allows the presence of a realistic minimum describing our present Universe with an infinitesimal positive vacuum energy arising due to a

${ }^{1}$ For reviews on supersymmetric models of inflation, see for example [1].

${ }^{2}$ See [3] for earlier work relating supersymmetry and inflation. 
cancellation between an F- and D-term contributions to the scalar potential, without affecting the properties of the inflationary plateau, along the lines of Refs. [5, 6, 7].

In the above models the D-term has a constant FI contribution but plays no role during inflation and can be neglected, while the pseudoscalar partner of the inflaton is absorbed by the $U(1)_{R}$ gauge field that becomes massive away from the origin. Recently, a new FI term was proposed [8] that has three important properties: (1) it is manifestly gauge invariant already at the Lagrangian level; (2) it is associated to a $U(1)$ that should not gauge an R-symmetry and (3) supersymmetry is broken by (at least) a D-auxiliary expectation value and the extra bosonic part of the action is reduced in the unitary gauge to a constant FI contribution leading to a positive shift of the scalar potential, in the absence of matter fields. In the presence of matter fields, the FI contribution to the D-term acquires a special field dependence $e^{2 K / 3}$ that violates invariance under Kähler transformations.

In a recent work [9], we studied the properties of the new FI term and explored its consequences to the class of inflation models we introduced in [4]. ${ }^{3}$ We first showed that matter fields charged under the $U(1)$ gauge symmetry can consistently be added in the presence of the new FI term, as well as a non-trivial gauge kinetic function. We then observed that the new FI term is not invariant under Kähler transformations. On the other hand, a gauged R-symmetry in ordinary Kähler invariant supergravity can always be reduced to an ordinary (non-R) $U(1)$ by a Kähler transformation. By then going to such a frame, we find that the two FI contributions to the $U(1)$ D-term can coexist, leading to a novel contribution to the scalar potential.

The resulting D-term scalar potential provides an alternative realisation of inflation from supersymmetry breaking, driven by a D- instead of an F-term. The inflaton is still a superpartner of the goldstino which is now a gaugino within a massive vector multiplet, where again the pseudoscalar partner is absorbed by the gauge field away from the origin. For a particular choice of the inflaton charge, the scalar potential has a maximum at the origin where inflation occurs and a supersymmetric minimum at zero energy, in the limit of negligible F-term contribution (such as in the absence of superpotential). The slow roll conditions are automatically satisfied near the point where the new FI term cancels the charge of the inflaton, leading to higher than quadratic contributions due to its non trivial field dependence.

The Kähler potential can be canonical, modulo the Kähler transformation that takes it to the non R-symmetry frame. In the presence of a small superpotential, the inflation is practically unchanged and driven by the D-term, as before. However, the maximum is now slightly shifted away from the origin and the minimum has a small non-vanishing positive vacuum energy, where supersymmetry is broken by both F- and D-auxiliary expectation values of similar magnitude. The model predicts in general small primordial gravitational waves with a tensor-to-scaler ration $r$ well below the observability limit. However, when higher order terms are included in the Kähler potential, one finds that $r$ can increase to large values $r \simeq 0.015$.

In the following, we will present the main features of these models, where inflation occurs near the maximum of the scalar potential where R-symmetry is restored and supersymmetry breaking is driven predominantly either by an F-term or by a D-term.

\section{INFLATION FROM SUPERSYMMETRY BREAKING}

\subsection{Set up}

Let us consider $D=4, N=1$ supergravity with one chiral multiplet transforming under a gauged R-symmetry with a corresponding abelian vector multiplet. We assume that the chiral multiplet $X$ transforms as:

$$
X \rightarrow e^{-i q \Lambda} X
$$

where $q$ is the charge of $X$, and $\Lambda$ is the gauge parameter. The Kähler potential is therefore a function of $X \bar{X}$ while the superpotential $\mathcal{W}$ is linear in $X$

$$
\mathcal{K}=\mathcal{K}(X \bar{X}), \quad \mathcal{W}=\kappa^{-3} f X,
$$

where $f$ is a constant. Note that $X$ is dimensionless and $\kappa^{-1}=2.4 \times 10^{18} \mathrm{GeV}$ is the reduced Planck mass. The gauge kinetic function is chosen to be 1 . The scalar potential is given by

$$
\mathcal{V}=\mathcal{V}_{F}+\mathcal{V}_{D}
$$

where the F and D-term potential are

$$
\mathcal{V}_{F}=e^{\kappa^{2} \mathcal{K}}\left(-3 \kappa^{2} \mathcal{W} \overline{\mathcal{W}}+\nabla_{X} \mathcal{W} g^{X} \bar{X} \bar{\nabla}_{\bar{X}} \overline{\mathcal{W}}\right), \quad \mathcal{V}_{D}=\frac{1}{2} \mathcal{P}^{2}
$$

The Kähler covariant derivative is acting on $\mathcal{W}$ as

$$
\nabla_{X} \mathcal{W}=\partial_{X} \mathcal{W}(z)+\kappa^{2}\left(\partial_{X} \mathcal{K}\right) \mathcal{W}
$$

The moment map $\mathcal{P}$ is given by

$$
\mathcal{P}=i\left(k^{X} \partial_{X} \mathcal{K}-r\right) .
$$

\footnotetext{
${ }^{3}$ This new FI term was also studied in [10] to remove an instability from inflation in Polonyi-Starobinsky supergravity.
} 
where $k^{X}$ is the Killing vector for $X$ under the $\mathrm{U}(1)$ R-symmetry, and $r$ is defined by $r=-\kappa^{-2} k^{X} \mathcal{W}_{X} / \mathcal{W}$; in the present setup, they become $k^{X}=-i q X, r=i \kappa^{-2} q$. As usual, subscripts stand for partial derivatives: $\mathcal{W}_{X}:=\partial_{X} \mathcal{W}$.

We are interested in the case where inflation starts near a local maximum of the potential at $X=0$, where R-symmetry is preserved. Let us expand the Kähler potential in $X \bar{X}$ up to quadratic order:

$$
\kappa^{2} \mathcal{K}=X \bar{X}+A(X \bar{X})^{2}+\ldots
$$

With this, the F-term potential becomes

$$
\kappa^{4} \mathcal{V}_{F}=f^{2} e^{X \bar{X}(1+A X \bar{X})}\left[-3 X \bar{X}+\frac{\left.\left(1+X \bar{X}+2 A(X \bar{X})^{2}\right)\right)^{2}}{1+4 A X \bar{X}}\right]
$$

and the D-term potential is

$$
\kappa^{4} \mathcal{V}_{D}=\frac{q^{2}}{2}\left[1+X \bar{X}+2 A(X \bar{X})^{2}\right]^{2}
$$

Under a change of field variables

$$
X=\rho e^{i \theta}, \quad \bar{X}=\rho e^{-i \theta}, \quad(\rho \geq 0)
$$

the scalar potential reads

$$
\begin{aligned}
\kappa^{4} \mathcal{V}= & f^{2} e^{\rho^{2}+A \rho^{4}}\left[-3 \rho^{2}+\frac{\left(1+\rho^{2}+2 A \rho^{4}\right)^{2}}{1+4 A \rho^{2}}\right] \\
& +\frac{q^{2}}{2}\left(1+\rho^{2}+2 A \rho^{4}\right)^{2}
\end{aligned}
$$

Note that the scalar potential is only a function of the modulus $\rho$ and the phase $\theta$ will be "eaten" by the $U(1)_{R}$ gauge field in a similar manner to the standard Brout-Englert-Higgs mechanism.

We now interpret the field $\rho$ as the inflaton. In order to calculate the slow-roll parameters, one needs to work with the canonically normalised field $\chi$ satisfying

$$
\frac{d \chi}{d \rho}=\sqrt{2 \mathcal{K}_{X \bar{X}}}
$$

The slow-roll parameters are given in terms of the canonical field $\chi$ by

$$
\epsilon=\frac{1}{2 \kappa^{2}}\left(\frac{d \mathcal{V} / d \chi}{\mathcal{V}}\right)^{2}, \quad \eta=\frac{1}{\kappa^{2}} \frac{d^{2} \mathcal{V} / d \chi^{2}}{\mathcal{V}}
$$

Since we assume inflation to start near $\rho=0$, we expand

$$
\begin{aligned}
& \epsilon=4\left(\frac{-4 A+y^{2}}{2+y^{2}}\right)^{2} \rho^{2}+\mathcal{O}\left(\rho^{4}\right) \\
& \eta=2\left(\frac{-4 A+y^{2}}{2+y^{2}}\right)+\mathcal{O}\left(\rho^{2}\right)
\end{aligned}
$$

where we defined $y=q / f$. The above equation implies $\epsilon \simeq \eta^{2} \rho^{2} \ll \eta$. For simplicity, we consider the case where the F-term potential is dominant by setting $y$ to be very small so that $y$ can be neglected. Taking this into account, let us find some constraints on the coefficient $A$ of the quadratic term of the Kähler potential. The condition that the scalar potential has a local maximum at $\rho=0$ requires $A>0$. Furthermore, the slow-roll condition $|\eta| \ll 1$ gives an upper bound $A \ll 0.25$. Therefore, the constraint on $A$ is

$$
0<A \ll 0.25
$$

In order to satisfy $\mathrm{CMB}$ observational data with $\eta \sim-0.02$, we choose $A \sim 0.005$. In the following sections we explore a microscopic model that can generate the coefficient $A$ satisfying the requirement (15).

\section{MICROSCOPIC MODEL}

In this section, we introduce a "generalisation" of the Fayet-Iliopoulos (FI) model as an example of the microscopic origin for the effective field theory of the inflation model described in the previous section. We consider the regime where both gauge symmetry and supersymmetry are spontaneously broken, leaving (in the decoupling limit) the goldstino as the only light mode in this sector. 


\subsection{The generalised Fayet-Iliopoulos model}

Let us consider a supergravity model with two chiral multiplets $\boldsymbol{\Phi}_{ \pm}$and one vector multiplet. As we will show shortly, this theory has a vacuum in which only $\boldsymbol{\Phi}_{+}$is lighter than the other degrees of freedom. We then integrate out the heavy degrees of freedom to find an effective supergravity action in $\boldsymbol{\Phi}_{+}$. The UV supergravity action we consider is

$$
\begin{aligned}
S= & \frac{1}{4} \int d^{4} x d^{2} \theta \mathcal{E} \mathcal{F}\left(\boldsymbol{\Phi}_{-}\right) \boldsymbol{W}^{\alpha} \boldsymbol{W}_{\alpha}+\text { h.c. } \\
& +\kappa^{-3} m \int d^{4} x d^{2} \theta \mathcal{E} \boldsymbol{\Phi}_{+} \boldsymbol{\Phi}_{-}+\text {h.c. } \\
& -3 \kappa^{-2} \int d^{4} x d^{4} \theta E e^{-\kappa^{2} \mathcal{K}_{0} / 3-\left(q_{+}-q_{-}\right) V / 3},
\end{aligned}
$$

which is formulated in Poincaré superspace as in [11]. In the following, we will mostly work in supergravity mass units $\kappa=1$, for notational simplicity. This theory is invariant under a gauged $U(1)$ transformation which acts only on matter superfields, which we call $\mathrm{U}(1)_{\mathrm{m}}$ transformation. The chiral superfields $\boldsymbol{\Phi}_{ \pm}$transform under $\mathrm{U}(1)_{\mathrm{m}}$ as, ${ }^{4}$

$$
\boldsymbol{\Phi}_{ \pm} \mapsto e^{\mp i q_{ \pm} \Lambda} \boldsymbol{\Phi}_{ \pm}
$$

where $\Lambda$ is chiral. The vector superfield $\boldsymbol{V}$ transforms under $\mathrm{U}(1)_{\mathrm{m}}$ as

$$
V \mapsto \boldsymbol{V}+i(\boldsymbol{\Lambda}-\bar{\Lambda})
$$

The function $\mathcal{K}_{0}$ is the $\mathrm{U}(1)_{\mathrm{m}}$-invariant Kähler potential,

$$
\kappa^{2} \mathcal{K}_{0}=\overline{\boldsymbol{\Phi}}_{+} e^{q_{+} V} \boldsymbol{\Phi}_{+}+\overline{\boldsymbol{\Phi}}_{-} e^{-q_{-} V} \boldsymbol{\Phi}_{-}
$$

and $\boldsymbol{W}_{\alpha}$ is the gaugino superfield, defined with the super-Poincaré covariant derivatives $\mathcal{D}_{\alpha}, \overline{\mathcal{D}}_{\dot{\alpha}}$ as

$$
W_{\alpha}=-\frac{1}{4} \overline{\mathcal{D}}^{2} \mathcal{D}_{\alpha} V
$$

The function $\mathcal{F}\left(\boldsymbol{\Phi}_{-}\right)$is the gauge kinetic function, given by

$$
\mathcal{F}\left(\boldsymbol{\Phi}_{-}\right)=1+b \ln \boldsymbol{\Phi}_{-}, \quad b=\frac{(x-1)^{3} q_{-}^{2}}{24 \pi^{2}}, \quad x=q_{+} / q_{-},
$$

in which the second term produces a Green-Schwarz action that cancels the chiral anomaly of $\mathrm{U}(1)_{\mathrm{m}}$. For more details see [4, 12].

The scalar potential of the UV theory (16) is given by

$$
\begin{aligned}
\kappa^{4} \mathcal{V}^{\mathrm{UV}}= & \frac{1}{4} q_{-}^{2} \frac{\left(x\left|\varphi_{+}\right|^{2}-\left|\varphi_{-}\right|^{2}+x-1\right)^{2}}{2(1+b \ln v)} \\
& \quad+m^{2} e^{\left|\varphi_{+}\right|^{2}+\left|\varphi_{-}\right|^{2}}\left(\left|\varphi_{+}\right|^{2}+\left|\varphi_{-}\right|^{2}-\left|\varphi_{+}\right|^{2}\left|\varphi_{-}\right|^{2}\right)
\end{aligned}
$$

where $\varphi_{ \pm}=\boldsymbol{\Phi}_{ \pm} \mid$is the lowest component of superfields $\boldsymbol{\Phi}_{ \pm}$. The first line is the D-term contribution. Note that it contains the Fayet-Iliopoulos type contribution with FI parameter $x-1$. Below, it is natural to introduce the parameter $\Delta$ as

$$
\Delta:=x-1-v^{2}
$$

We are interested in a vacuum of the form

$$
\left\langle\varphi_{+}\right\rangle=0, \quad\left\langle\varphi_{-}\right\rangle=v,
$$

which spontaneously breaks $\mathrm{U}(1)_{\mathrm{m}}$ and supersymmetry and around which the fields of $\boldsymbol{V}, \boldsymbol{\Phi}_{-}$are heavier than those of $\boldsymbol{\Phi}_{+}$, in the limit of small SUSY breaking scale. The extremisation condition with respect to $\varphi_{-}$reads

$$
-\frac{1}{4} q_{-}^{2} \frac{\Delta}{1+b \ln v} v^{2}-\frac{1}{16} b q_{-}^{2}\left(\frac{\Delta}{1+b \ln v}\right)^{2}+m^{2} v^{2}\left(1+v^{2}\right) e^{v^{2}}=0 .
$$

This gives us a constraint among the parameters $\Delta, v, x$ and $q_{-}$which will be used in Section 3.4.

\footnotetext{
${ }^{4}$ Strictly speaking, it involves a local rotation of the fermionic coordinates in addition to the overall phase rotation, due to the non-invariance of the superpotential
} under $\mathrm{U}(1)_{\mathrm{m}}$. In this sense, $\mathrm{U}(1)_{\mathrm{m}}$ is a gauged R-transformation, which is allowed only in supergravity. 
We consider first the approximation $b=0$. In this case equation (25) has a unique solution

$$
\Delta=\frac{4 m^{2}}{q_{-}^{2}}\left(1+v^{2}\right) e^{v^{2}}
$$

It is also easy to see that $\operatorname{Im} \varphi_{-}$is the massless R-Goldstone boson while $\operatorname{Re} \varphi_{-}$gets a correction to its mass-squared compared to the global SUSY case $q_{-}^{2} v^{2}$ by $4 m^{2} v^{2}\left(2+v^{2}\right) e^{v^{2}}$. The mass-squared of $\varphi_{+}$also changes to $m^{2}\left(1+x+x v^{2}\right) e^{v^{2}}$ and the integrating out condition is satisfied if this mass is much smaller than the other masses.

For $b \neq 0$ eq. (25) gives two solutions $\Delta=\Delta_{ \pm}$, where $\Delta_{ \pm}$are given by

$$
\Delta_{ \pm}:=\frac{2 v^{2}(1+b \ln v)}{b}\left(-1 \pm \sqrt{1+\frac{4 b m^{2}\left(1+v^{2}\right) e^{v^{2}}}{q_{-}^{2} v^{2}}}\right)
$$

Notice that the existence of the two solutions originates from the anomaly coefficient $b$. The mass ${ }^{2}$ of the vector field $A_{\mu}$ is $q_{-}^{2} v^{2}$. The mass matrices of $\varphi_{ \pm}$are given by

$$
\begin{aligned}
& \left.\mathcal{V}_{\varphi_{+}^{*} \varphi_{+}^{*}}^{\mathrm{UV}}\right|_{\mathrm{vac}}=0, \\
& \left.\mathcal{V}_{\varphi_{+}^{*} \varphi_{+}}^{\mathrm{UV}}\right|_{\mathrm{vac}}=m^{2} e^{v^{2}}+\frac{1}{4} x q_{-}^{2} \frac{\Delta}{1+b \ln v^{\prime}}, \\
& \left.\mathcal{V}_{\varphi_{-}^{*} \varphi_{-}^{*}}^{\mathrm{UV}}\right|_{\mathrm{vac}}=m^{2} e^{v^{2} v^{2}}\left(2+v^{2}\right)+\frac{1}{4} q_{-}^{2} \frac{v^{2}}{1+b \ln v} \\
& +\frac{1}{16} b q_{-}^{2} \frac{\Delta^{2}}{v^{2}(1+b \ln v)^{2}}+\frac{1}{4} b q_{-}^{2} \frac{\Delta}{(1+b \ln v)^{2}} \\
& +\frac{1}{16} b^{2} q_{-}^{2} \frac{\Delta^{2}}{v^{2}(1+b \ln v)^{3}} \\
& \left.\mathcal{V}_{\varphi_{-}^{*} \varphi_{-}}^{\mathrm{UV}}\right|_{\mathrm{vac}}=m^{2} e^{v^{2}}\left(1+3 v^{2}+v^{4}\right)+\frac{1}{4} q_{-}^{2} \frac{v^{2}}{1+b \ln v} \\
& -\frac{1}{4} q_{-}^{2} \frac{\Delta}{1+b \ln v}+\frac{1}{4} b q_{-}^{2} \frac{\Delta}{(1+b \ln v)^{2}} \\
& +\frac{1}{16} b^{2} q_{-}^{2} \frac{\Delta^{2}}{v^{2}(1+b \ln v)^{3}} \text {. }
\end{aligned}
$$

In the following, we assume that the integrating out procedure is justified, which we will show explicitly in Section 3.4 with the analysis of the parameter space leading to models of realistic inflation.

\subsection{Integrating out heavy fields}

The UV action in conformal superspace which becomes the action (16) after fixing the conformal compensators as $C=\bar{C}=1$ is actually very easy to write down,

$$
\begin{aligned}
S= & \frac{1}{4} \int d^{4} x d^{2} \theta \mathcal{E} \mathcal{F}\left(\boldsymbol{\Phi}_{-}\right) \boldsymbol{W}^{\alpha} \boldsymbol{W}_{\alpha}+\text { h.c. } \\
& +\kappa^{-3} m \int d^{4} x d^{2} \theta \mathcal{E} C^{3} \boldsymbol{\Phi}_{+} \boldsymbol{\Phi}_{-}+\text {h.c. } \\
& -3 \kappa^{-2} \int d^{4} x d^{4} \theta \boldsymbol{E} C \overline{\boldsymbol{C}} e^{-\kappa^{2} \mathcal{K}_{0} / 3-\left(q_{+}-q_{-}\right) V / 3},
\end{aligned}
$$

and takes exactly the same form as in the case with the super-Weyl compensators [13]. To explain the notation, we need to introduce two important classes of superfields in conformal superspace: chiral and primary. A chiral superfield $\boldsymbol{\Phi}$ is defined with respect to the superconformally covariant spinor derivative $\bar{\nabla}_{\dot{\alpha}}$ by

$$
\bar{\nabla}_{\dot{\alpha}} \boldsymbol{\Phi}=0 .
$$

A primary superfield $\boldsymbol{\Phi}$ of charges $(\delta, \tilde{w})$ (conformal and chiral weights) is defined by

$$
\hat{D} \boldsymbol{\Phi}=\delta \boldsymbol{\Phi}, \quad \hat{A} \boldsymbol{\Phi}=i \tilde{w} \Phi, \quad \hat{K}_{A} \boldsymbol{\Phi}=0,
$$

where $\hat{D}, \hat{A}, \hat{K}_{A}$ are the generators for the dilatation, chiral $\mathrm{U}(1)$ rotation, and special conformal transformations [14]. ${ }^{5}$

\footnotetext{
${ }^{5}$ The local Lorentz index $A$ in $\hat{K}_{A}$ stands for the vector and the undotted and dotted spinor indices $(a, \alpha, \dot{\alpha})$. Therefore $\hat{K}_{A}$ denotes the generators $\left(\hat{K}_{a}, \hat{S}_{\alpha}, \hat{S}^{\dot{\alpha}}\right)$, with $\hat{S}_{\alpha}$, $\hat{S}^{\dot{\alpha}}$ the generators of the S-supersymmetry.
} 
We now explain the notation. For details, see [15]. An action integral with $\int d^{4} x d^{4} \theta$ like the third line of (32) is called the D-type action. Its integrand is required to be real primary of charge $(0,0)$ for gauge invariance. On the other hand, an action integral with $\int d^{4} x d^{2} \theta$ like the first and second lines of (32) is called the F-type action. Its integrand is required to be chiral primary of charge $(0,0)$ for gauge invariance.

The determinant $E$ of the vierbein superfield is real primary of charges $(-2,0)$, while the determinant $\mathcal{E}$ of the "chiral" part of the vierbein superfield, called the chiral density, is chiral primary of charges $(-3,-2)$.

The chiral superfields $\boldsymbol{\Phi}_{ \pm}$are primaries of charges $(0,0)$, transforming under the matter $\mathrm{U}(1) \mathrm{m}$ as $\boldsymbol{\Phi}_{ \pm} \mapsto e^{\mp i q_{ \pm}} \boldsymbol{\Lambda} \boldsymbol{\Phi}_{ \pm}$, where $\boldsymbol{\Lambda}$ is chiral primary of charges $(0,0)$. The vector superfield $\boldsymbol{V}$ is primary of charges $(0,0)$, which transforms under $\mathrm{U}(1)_{\mathrm{m}}$ as $\boldsymbol{V} \mapsto$ $\boldsymbol{V}+i(\boldsymbol{\Lambda}-\overline{\boldsymbol{\Lambda}})$.

The compensators $C, \bar{C}$ are chiral primaries of charges $(1,2 / 3)$, and anti-chiral primary of charges $(1,-2 / 3)$, respectively. To guarantee $\mathrm{U}(1)_{\mathrm{m}}$ invariance, we need to assign $\mathrm{U}(1)_{\mathrm{m}}$ charges to the compensators $C, \bar{C}$ as

$$
C \mapsto e^{i\left(q_{+}-q_{-}\right) \Lambda / 3} C, \quad \bar{C} \mapsto e^{-i\left(q_{+}-q_{-}\right) \bar{\Lambda} / 3} \bar{C} .
$$

$\mathcal{K}_{0}$ is the gauge-invariant Kähler potential defined in (19) and $\boldsymbol{W}_{\alpha}$ is the chiral primary gaugino superfield of charges $(3 / 2,1)$, defined here with the superconformally covariant derivatives $\nabla_{\alpha}, \bar{\nabla}_{\dot{\alpha}}$ as ${ }^{6}$

$$
\boldsymbol{W}_{\alpha}=-\frac{1}{4} \bar{\nabla}^{2} \nabla_{\alpha} \boldsymbol{V}
$$

We proceed to integrating out the heavy degrees of freedom. For this, we first fix the matter $\mathrm{U}(1)_{\mathrm{m}}$ degrees of freedom by the unitary gauge $\boldsymbol{\Phi}_{-}=v$, in which the action reads

$$
\begin{aligned}
S= & \frac{1}{4} \int d^{4} x d^{2} \theta \mathcal{E} \boldsymbol{W}^{\alpha} \boldsymbol{W}_{\alpha}+\text { h.c. } \\
& +\kappa^{-3} m v \int d^{4} x d^{2} \theta \mathcal{E} C^{3} \boldsymbol{\Phi}_{+}+\text {h.c. } \\
& -3 \kappa^{-2} \int d^{4} x d^{4} \theta \boldsymbol{E} C \overline{\boldsymbol{C}} e^{-\kappa^{2} \mathcal{K} / 3},
\end{aligned}
$$

where we rescaled $V$ to absorb the factor $1+b \ln v$, and $\mathcal{K}$ is the gauge-fixed Kähler potential with the FI contribution,

$$
\kappa^{2} \mathcal{K}=\overline{\boldsymbol{\Phi}}_{+} e^{x q_{-} \boldsymbol{V}} \boldsymbol{\Phi}_{+}+v^{2} e^{-q_{-} \boldsymbol{V}}+(x-1) q_{-} \boldsymbol{V}
$$

and we recall $x=q_{+} / q_{-}$.

We integrate out $V$ at tree level by solving the equation of motion of $V$ around its vacuum, neglecting higher derivative contributions. The equation of motion of $V$ reads

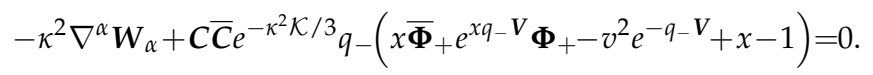

To integrate out $V$ around its vacuum, we first shift $\nabla^{\alpha} \boldsymbol{W}_{\alpha} \mid$ to remove the tadpole [16], and then neglect the derivative term. This gives the following low-energy effective equation of motion

$$
C \overline{\boldsymbol{C}} e^{-\kappa^{2} \mathcal{K} / 3} q_{-}\left(x \overline{\boldsymbol{\Phi}}_{+} e^{x q_{-} V} \boldsymbol{\Phi}_{+}-v^{2} e^{-q_{-} \boldsymbol{V}}+x-1\right)-q_{-} \Delta \simeq 0 .
$$

Recall that $\Delta=x-1-v^{2}$. We now integrate out $V$ in the following way:

$$
\frac{1}{4} \int d^{4} x d^{2} \theta \mathcal{E} \boldsymbol{W}^{\alpha} \boldsymbol{W}_{\alpha}+\text { h.c. }=-\frac{1}{2} \int d^{4} x d^{4} \theta \boldsymbol{E} \boldsymbol{V} \nabla^{\alpha} \boldsymbol{W}_{\alpha},
$$

and then eliminate $\nabla^{\alpha} \boldsymbol{W}_{\alpha}$ by substituting the exact equation of motion (39). The first and third terms of the action (37) then become

$$
\begin{aligned}
& \int d^{4} x d^{4} \theta \boldsymbol{E}\left(-\frac{1}{2} \boldsymbol{V} \nabla^{\alpha} \boldsymbol{W}_{\alpha}-3 \kappa^{-2} \boldsymbol{C} \overline{\boldsymbol{C}} e^{-\kappa^{2} \mathcal{K} / 3}\right) \\
= & \kappa^{-2} \int d^{4} x d^{4} \theta \boldsymbol{E} \boldsymbol{C} \overline{\boldsymbol{C}} e^{-\kappa^{2} \mathcal{K} / 3} \\
& \times\left[-\frac{1}{2} q_{-} \boldsymbol{V}\left(x \overline{\boldsymbol{\Phi}}_{+} e^{x q_{-} \boldsymbol{V}} \boldsymbol{\Phi}_{+}-v^{2} e^{-q_{-} \boldsymbol{V}}+x-1\right)-3\right] .
\end{aligned}
$$

Next, combining the (low-energy) equation of motion (40) with the second line of (42), we obtain the low-energy effective action,

$$
\begin{aligned}
S_{\text {eff }}\left[\boldsymbol{C} ; \boldsymbol{\Phi}_{+}\right]= & \kappa^{-3} m v \int d^{4} x d^{2} \theta \mathcal{E} C^{3} \boldsymbol{\Phi}_{+}+\text {h.c. } \\
& +\kappa^{-2} \int d^{4} x d^{4} \theta \boldsymbol{E}\left(-\frac{1}{2} \Delta q-\boldsymbol{V}-3 \boldsymbol{C} \overline{\boldsymbol{C}} e^{-\kappa^{2} \mathcal{K} / 3}\right),
\end{aligned}
$$

where $\boldsymbol{V}$ must be understood to be a function of $\boldsymbol{\Phi}_{+}$, determined by the equation of motion (40).

\footnotetext{
${ }^{6}$ Note that $\nabla_{\alpha}$ has charges $(1 / 2,-1)$ and $\bar{\nabla}_{\dot{\alpha}}$ has charges $(1 / 2,1)$.
} 


\begin{tabular}{c|c|c} 
& $\mathrm{U}(1)_{m}$ & $\mathrm{U}(1)^{\prime}$ \\
\hline $\boldsymbol{\Phi}_{+}$ & $+q_{+}$ & $q$ \\
\hline $\boldsymbol{\Phi}_{-}$ & $-q_{-}$ & 0
\end{tabular}

TABLE 1: The chiral multiplet $\boldsymbol{\Phi}_{+}$and $\boldsymbol{\Phi}_{-}$are charged under $\mathrm{U}(1)_{m} \times \mathrm{U}(1)^{\prime}$. Note that $\mathrm{U}(1)^{\prime}$ does not play any role during the integrating out process and remains R-symmetry of the low-energy theory.

\subsection{Effective Kähler potential and superpotential}

Let us now fix the compensators. We find $\mathcal{K}_{\text {eff }}$ such that the gauge fixing

$$
C=\bar{C}=e^{\kappa^{2} \mathcal{K}_{\text {eff }} / 6}
$$

makes the effective action (43) into the integral in the Kähler superspace characterised by $\mathcal{K}_{\text {eff. }}$ It is easy to see that this is realised by

$$
\kappa^{2} \mathcal{K}_{\text {eff }}=\kappa^{2} \mathcal{K}+3 \ln \left(1-\frac{1}{6} \Delta q_{-} \boldsymbol{V}\right), \quad \kappa^{3} \mathcal{W}_{\text {eff }}=m v \boldsymbol{\Phi}_{+} .
$$

The second term in the effective Kähler potential is the supergravity modification. The gauge fixing (44) simplifies the effective equation of motion (40) into

$$
\left(1-\frac{1}{6} \Delta q_{-} \boldsymbol{V}\right)\left(x \overline{\boldsymbol{\Phi}}_{+} e^{x q_{-} \boldsymbol{V}} \boldsymbol{\Phi}_{+}-v^{2} e^{-q_{-} \boldsymbol{V}}+x-1\right)-\Delta=0,
$$

which can be solved analytically for $\overline{\boldsymbol{\Phi}}_{+} \boldsymbol{\Phi}_{+}$as a function of $V$,

$$
\begin{aligned}
\overline{\boldsymbol{\Phi}}_{+} \boldsymbol{\Phi}_{+} & =x^{-1} e^{-x q_{-} \boldsymbol{V}}\left(v^{2} e^{-q_{-} \boldsymbol{V}}-x+1+\frac{\Delta}{1-\frac{1}{6} \Delta q_{-} \boldsymbol{V}}\right) \\
& =x^{-1} e^{-x q_{-} \boldsymbol{V}}\left(v^{2} e^{-q_{-} \boldsymbol{V}}-v^{2}+\frac{\frac{1}{6} \Delta^{2} q_{-} \boldsymbol{V}}{1-\frac{1}{6} \Delta q_{-} \boldsymbol{V}}\right) .
\end{aligned}
$$

The effective theory found in the this section does not have a gauged $\mathrm{R}$ symmetry. Therefore, we need to add another gauged $\mathrm{R}$ symmetry to the low-energy theory, which we denote by $\mathrm{U}(1)^{\prime}$. This can be achieved by extending the symmetry of the UV theory from $\mathrm{U}(1)_{\mathrm{m}}$ to $\mathrm{U}(1)_{\mathrm{m}} \times \mathrm{U}(1)^{\prime}$. We assume that $\mathrm{U}(1)^{\prime}$ acts as a spectator during the integrating out process and survives as the gauged R-symmetry of the low-energy theory. As summarised in Table $1, \boldsymbol{\Phi}_{+}$transforms under $\mathrm{U}(1)_{m} \times \mathrm{U}(1)^{\prime}$ with charge $\left(q_{+}, q\right)$ while $\boldsymbol{\Phi}_{-}$is singlet under $\mathrm{U}(1)^{\prime}$.

In what follows, we will analyse the behaviour of the effective Kähler potential around the origin and identify the parameter regions in which the scalar potential has a local maximum at the origin.

\subsection{Inflation from the effective low-energy theory}

For simplicity, we absorb $q_{-}$into the vector multiplet. ${ }^{7}$ To obtain the behaviour around the origin, we should first solve for $V$ in terms of $\overline{\boldsymbol{\Phi}}_{+} \boldsymbol{\Phi}_{+}$from equation (46) perturbatively in the form

$$
\boldsymbol{V}=V_{0}+V_{1} \overline{\boldsymbol{\Phi}}_{+} \boldsymbol{\Phi}_{+}+V_{2}\left(\overline{\boldsymbol{\Phi}}_{+} \boldsymbol{\Phi}_{+}\right)^{2}+V_{3}\left(\overline{\boldsymbol{\Phi}}_{+} \boldsymbol{\Phi}_{+}\right)^{3}+\ldots .
$$

Substituting this into equation (46) we obtain an explicit expression for the coefficients,

$$
\begin{aligned}
V_{0}= & 0, \quad V_{1}=\frac{6 x}{\Delta^{2}-6 v^{2}}, \\
V_{2}= & \frac{6 x^{2}}{\left(\Delta^{2}-6 v^{2}\right)^{3}}\left(-\Delta^{3}+6 \Delta^{2} x-18 v^{2}(2 x+1)\right), \\
V_{3}= & \frac{6 x^{3}}{\left(\Delta^{2}-6 v^{2}\right)^{5}}\left(\Delta^{6}-18 \Delta^{5} x+6 \Delta^{4}\left(v^{2}+9 x^{2}\right)+36 \Delta^{3} v^{2}(3 x+2)\right. \\
& \left.-36 \Delta^{2} v^{2}\left(18 x^{2}+9 x-1\right)+216 v^{4}\left(18 x^{2}+9 x+2\right)\right) .
\end{aligned}
$$

Substituting the perturbative solution (48) into the effective Kähler potential (45), we obtain the effective Kähler potential around the local maximum,

$$
\kappa^{2} \mathcal{K}_{\text {eff }}=v^{2}+K_{1} \overline{\boldsymbol{\Phi}}_{+} \boldsymbol{\Phi}_{+}+K_{2}\left(\overline{\boldsymbol{\Phi}}_{+} \boldsymbol{\Phi}_{+}\right)^{2}+K_{3}\left(\overline{\boldsymbol{\Phi}}_{+} \boldsymbol{\Phi}_{+}\right)^{3}+\ldots,
$$

\footnotetext{
${ }^{7}$ More precisely, we first rescale $q_{-}$as $q_{-} \rightarrow q_{-}(1+b \ln v)^{-1 / 2}$ and then rescale $V$ as $q_{-} V \rightarrow V$ with the rescaled $q_{-}$in the unitary gauge action (37).
} 
where the first three coefficients read

$$
\begin{aligned}
K_{1}= & \frac{\Delta^{2}+3 \Delta x-6 v^{2}}{\Delta^{2}-6 v^{2}} \\
K_{2}= & -\frac{3 x^{2}\left(-\Delta^{4}-12 \Delta^{3} x+30 \Delta^{2} v^{2}+36 \Delta v^{2}(2 x+1)-72 v^{4}\right)}{2\left(\Delta^{2}-6 v^{2}\right)^{3}}, \\
K_{3}= & \frac{x^{3}}{\left(\Delta^{2}-6 v^{2}\right)^{5}}\left\{-\Delta^{7}-18 \Delta^{6} x+6 \Delta^{5}\left(8 v^{2}+27 x^{2}\right)-18 \Delta^{4} v^{2}(12 x-7)\right. \\
& -36 \Delta^{3} v^{2}\left(v^{2}+54 x^{2}+27 x-3\right)+108 \Delta^{2} v^{4}(24 x+7) \\
& \left.+648 \Delta v^{4}\left(9 x^{2}+9 x+2\right)-1296 v^{6}(3 x+1)\right\} .
\end{aligned}
$$

We then define the canonically normalized chiral superfield $\boldsymbol{\Phi}$ as

$$
\boldsymbol{\Phi}:=\sqrt{K_{1}} \boldsymbol{\Phi}_{+}
$$

After absorbing the constant term $v^{2}$ in (50) by a Kähler transformation, the effective Kähler potential in $\boldsymbol{\Phi}$ becomes

$$
\kappa^{2} \mathcal{K}_{\text {eff }}=\overline{\boldsymbol{\Phi}} \boldsymbol{\Phi}+A_{2}(\overline{\boldsymbol{\Phi}} \boldsymbol{\Phi})^{2}+A_{3}(\overline{\boldsymbol{\Phi}} \boldsymbol{\Phi})^{3}+\ldots,
$$

where the first two nontrivial coefficients $A_{2}, A_{3}$ read

$$
\begin{aligned}
A_{2}= & \frac{3 x^{2}\left(\Delta^{4}+12 \Delta^{3} x-30 \Delta^{2} v^{2}-36 \Delta v^{2}(2 x+1)+72 v^{4}\right)}{2\left(\Delta^{2}-6 v^{2}\right)\left(\Delta^{2}+3 \Delta x-6 v^{2}\right)^{2}} \\
A_{3}= & \frac{x^{3}}{\left(\Delta^{2}-6 v^{2}\right)^{2}\left(\Delta^{2}+3 \Delta x-6 v^{2}\right)^{3}}\left\{-\Delta^{7}-18 \Delta^{6} x\right. \\
& +6 \Delta^{5}\left(8 v^{2}+27 x^{2}\right)+18 \Delta^{4} v^{2}(7-12 x) \\
& -36 \Delta^{3} v^{2}\left(v^{2}+54 x^{2}+27 x-3\right)+108 \Delta^{2} v^{4}(24 x+7) \\
& \left.+648 \Delta v^{4}\left(18 x^{2}+9 x+2\right)-1296 v^{6}(3 x+1)\right\}
\end{aligned}
$$

The condition for having a local maximum at the origin is $A_{2}>0$. In the two-dimensional parameter space $(v, x)$, the domain in which $A_{2}$ is positive can be divided into four regions according to the signs of $\Delta=x-1-v^{2}$ and of the scalar component $c=V \mid$ in each region. They are

- Region I: with $\Delta>0, c \geqslant 0$,

- Region II: with $\Delta>0, c \leqslant 0$,

- Region III: with $\Delta<0, c \leqslant 0$,

- Region IV: with $\Delta<0, c \geqslant 0$.

These four regions are shown in Fig. 1a. It turns out that a Minkowski minimum is allowed in the presence of D-term in Region I and III, while Region II and IV have only de Sitter minimum with a large cosmological constant. The integrating out condition also excludes Region I. Therefore, this leaves Region III as the only possible domain for slow-roll inflation with a nearby minimum having a tuneable vacuum energy.

\subsection{The effective scalar potential and slow-roll parameters}

In order to study the global minimum of the potential and compare our predictions for inflation with the observational data, we need the exact expression of the scalar potential. Using the analytic solution (47) for $\overline{\boldsymbol{\Phi}}_{+} \boldsymbol{\Phi}_{+}$as a function of $\boldsymbol{V}$, we will express the scalar potential as a function of $c=V \mid$ instead of $\varphi_{+}=\boldsymbol{\Phi}_{+} \mid$.

Combining the effective Kähler potential (45) with the analytic solution (47), we express the effective Kähler potential as a function of the vector multiplet $V$,

$$
\begin{aligned}
\kappa^{2} \mathcal{K}_{\text {eff }}(\boldsymbol{V})=\frac{1}{x} & {\left[v^{2}(1+x) e^{-\boldsymbol{V}}+\frac{\Delta}{1-\frac{1}{6} \Delta \boldsymbol{V}}-x+1\right]+(x-1) \boldsymbol{V} } \\
& +3 \ln \left[1-\frac{1}{6} \Delta \boldsymbol{V}\right] .
\end{aligned}
$$




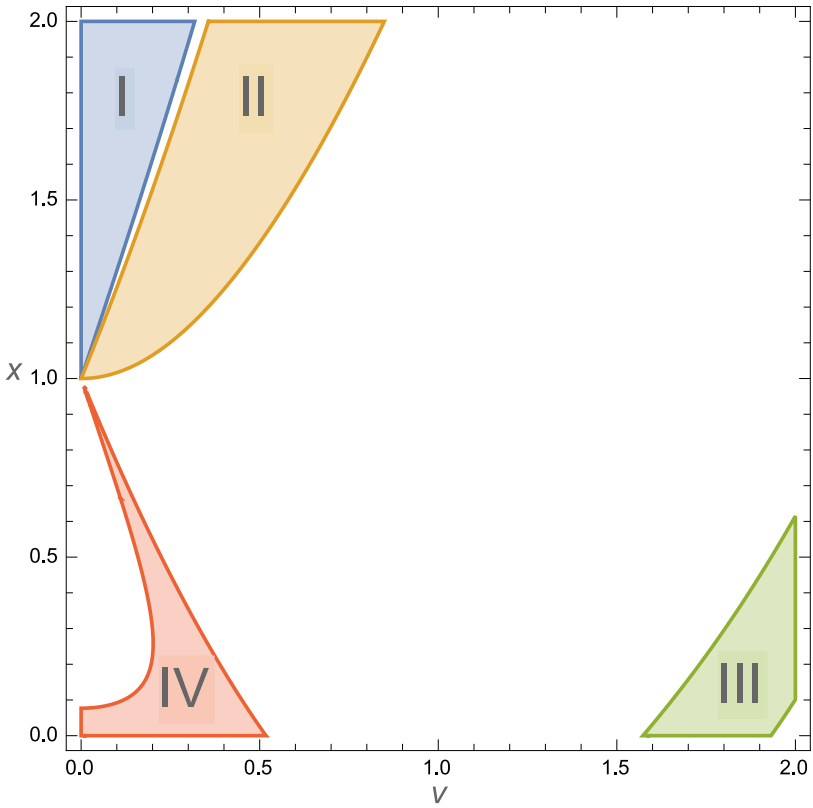

(a)

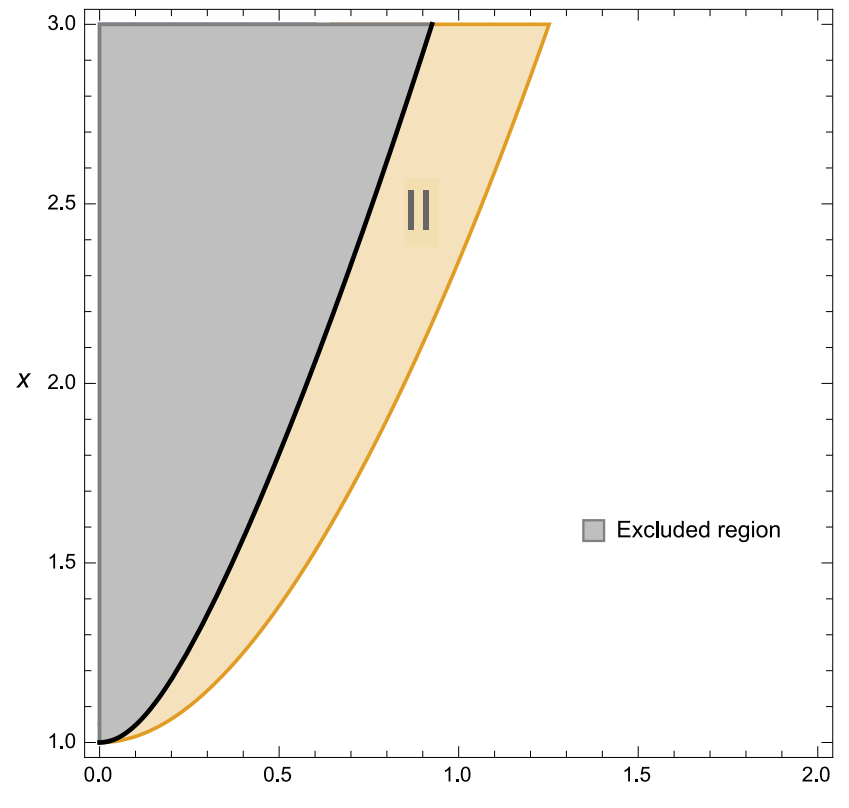

(b)

FIGURE 1: (a) Allowed parameter space $(v, x)$ with $0<v<2.0$ and $0<x<2.0$. The colored regions in which $A_{2}>0$ can be divided into 4 parts, namely I, II, III and IV. (b) Region I and part of Region II are in the excluded area where $v^{2}-\frac{1}{4} x\left(x-1-v^{2}\right)<0$ where the integrating out condition is not satisfied.

Note that $V$ must be understood as a function of $\overline{\boldsymbol{\Phi}}_{+} \boldsymbol{\Phi}_{+}$. The effective superpotential is

$$
\kappa^{3} \mathcal{W}_{\text {eff }}=m v \Phi_{+}
$$

Using the formula (4) and expressing it in the D-term potential in terms of $c=V \mid$ instead of $\varphi_{+}=\boldsymbol{\Phi}_{+} \mid$, we find the low energy D-term potential given by

$$
\begin{aligned}
\kappa^{4} \mathcal{V}_{D}=\frac{y^{2} e^{-2 c} m^{2} v^{2}}{8 x^{2}} & {\left[\rho v^{2}\left(x+1-x e^{c}\right) c^{\prime}-2 e^{c} x\right.} \\
& \left.-e^{c} \rho c^{\prime} \frac{x \Delta(3-c \Delta)}{6-c \Delta}-\frac{6 e^{c} \rho c^{\prime} \Delta^{2}}{(6-c \Delta)^{2}}\right]^{2},
\end{aligned}
$$

where we introduced a new parameter $y:=q / m v$. Recall also that $\Delta=x-1-v^{2}$. The new field variable $\rho$ is defined as $\rho:=$ $\left(\varphi_{+}^{*} \varphi_{+}\right)^{1 / 2}$, which stands for the inflaton. This can be written in terms of $c$ with the help of (47) as

$$
\rho^{2}=\frac{e^{-x c}}{x}\left[v^{2} e^{-c}-x+1+\frac{\Delta}{1-\frac{1}{6} \Delta c}\right] .
$$

For any given value of the parameters $v$ and $x$, we can choose the "physical domain" of $c$ in such a way that $\rho^{2}>0$. We also introduced $c^{\prime}=d c / d \rho, c^{\prime \prime}=d^{2} c / d \rho^{2}$, which can be expressed in terms of $c$ with the help of (61) as

$$
\begin{gathered}
c^{\prime}=\frac{2 \rho x(6-c \Delta) e^{c(x+1)}}{e^{c} \Delta^{2}-v^{2}\left(6-\left(c+e^{c}-1\right) \Delta\right)-\rho^{2} x e^{c(x+1)}(6 x-c \Delta x-\Delta)}, \\
c^{\prime \prime}=-\frac{v^{2}(6-c \Delta+2 \Delta)\left(c^{\prime}\right)^{2}}{e^{c} \Delta^{2}-v^{2}\left(6-\left(c+e^{c}-1\right) \Delta\right)-\rho^{2} x e^{c(x+1)}(6 x-c \Delta x-\Delta)} \\
+\frac{x e^{c(x+1)}\left(\rho c^{\prime}\left(\rho x c^{\prime}(x(6-c \Delta)-2 \Delta)+4(x(6-c \Delta)-\Delta)\right)+2(6-c \Delta)\right)}{e^{c} \Delta^{2}-v^{2}\left(6-\left(c+e^{c}-1\right) \Delta\right)-\rho^{2} x e^{c(x+1)}(6 x-c \Delta x-\Delta)}
\end{gathered}
$$

On the other hand, the effective F-term potential is given by

$$
\kappa^{4} \mathcal{V}_{F}=m^{2} v^{2} e^{\kappa^{2} \mathcal{K}_{\text {eff }}(c)}\left(-3 \rho^{2}+\frac{4 \mathcal{A}^{2}(c)}{\mathcal{B}(c)}\right)
$$


where we introduced two functions $\mathcal{A}(c), \mathcal{B}(c)$

$$
\begin{aligned}
\mathcal{A}(c)= & 1+\frac{\rho c^{\prime}}{2 x(6-c \Delta)^{2}} e^{-c}\left[6 e^{c} v^{4}+e^{c} \xi\left(x\left(c^{2} \Delta^{2}-9 c \Delta+18\right)+6 \xi\right)\right. \\
& \left.+v^{2}\left(-c^{2} \Delta^{2}+12 c \Delta-12 e^{c} \xi+x(6-c \Delta)\left(c \Delta+3 e^{c}-6\right)-36\right)\right], \\
\mathcal{B}(c)= & -\frac{3 \Delta\left(\rho c^{\prime \prime}+c^{\prime}\right)}{\rho(6-c \Delta)}+\frac{\xi\left(\rho c^{\prime \prime}+c^{\prime}\right)}{\rho} \\
& +\frac{\left(\rho c^{\prime \prime}+c^{\prime}\right)\left(\frac{6 \Delta^{2}}{(6-c \Delta)^{2}}-e^{-c} v^{2}(x+1)\right)}{x \rho} \\
& +\frac{\left(c^{\prime}\right)^{2}}{x}\left(e^{-c} v^{2}(x+1)+\frac{12 \Delta^{3}}{(6-c \Delta)^{3}}\right)-\frac{3 \Delta^{2}\left(c^{\prime}\right)^{2}}{(6-c \Delta)^{2}} .
\end{aligned}
$$

To compute the slow-roll parameters, we need the canonically normalised inflaton field $\chi$ defined through $\chi^{\prime}:=\frac{d \chi}{d \rho}=$ $\sqrt{2 g_{\overline{\boldsymbol{\Phi}}_{+} \boldsymbol{\Phi}_{+}}}$, which can be written in terms of $c$ as

$$
\chi^{\prime}=\kappa \sqrt{\left(\frac{c^{\prime}}{2 \rho}+\frac{c^{\prime \prime}}{2}\right) \frac{d}{d c} \mathcal{K}_{\mathrm{eff}}(c)+\frac{\left(c^{\prime}\right)^{2}}{2 \rho} \frac{d^{2}}{d c^{2}} \mathcal{K}_{\mathrm{eff}}(c) .}
$$

The slow-roll parameters $\epsilon$ and $\eta$ are given in terms of $c$ by

$$
\begin{aligned}
\epsilon & =\frac{1}{2 \kappa^{2}}\left(\frac{d \mathcal{V} / d \chi}{\mathcal{V}}\right)^{2}=\frac{1}{2 \kappa^{2}}\left(\frac{d \mathcal{V} / d c}{\mathcal{V}} \frac{c^{\prime}}{\chi^{\prime}}\right)^{2}, \\
\eta & =\frac{1}{\kappa^{2}} \frac{d^{2} \mathcal{V} / d \chi^{2}}{\mathcal{V}}, \\
& =\frac{1}{\kappa^{2}}\left(\frac{d^{2} \mathcal{V} / d c^{2}}{\mathcal{V}}\left(\frac{c^{\prime}}{\chi^{\prime}}\right)^{2}+\frac{d \mathcal{V} / d c}{\mathcal{V}} \frac{c^{\prime \prime}}{\chi^{\prime}}-\frac{d \mathcal{V} / d c}{\mathcal{V}} \frac{d \chi^{\prime} / d c}{\chi^{\prime}}\left(\frac{c^{\prime}}{\chi^{\prime}}\right)^{2}\right)
\end{aligned}
$$

The number of e-folds $N$ during inflation can be expressed as

$$
N=\int_{\chi_{*}}^{\chi_{\text {end }}} \frac{\mathcal{V}}{\partial_{\chi} \mathcal{V}} d \chi=\int_{\rho_{*}}^{\rho_{\text {end }}} \frac{\mathcal{V}}{\partial_{\rho} \mathcal{V}}\left(\chi^{\prime}\right)^{2} d \rho=\int_{c_{\text {end }}}^{c_{*}} \frac{\mathcal{V}}{\partial_{\mathcal{C}} \mathcal{V}}\left(\frac{\chi^{\prime}}{c^{\prime}}\right)^{2} d c,
$$

where we choose $\left|\eta\left(c_{\text {end }}\right)\right|=1$ and $c_{*}$ is the value of $c$ at the horizon exit. Now we can compare the theoretical predictions of our model to the observational data, specifically the power spectrum of primordial perturbations of the CMB, namely the amplitude of density fluctuations $A_{s}$, the spectral index $n_{s}$ and the tensor-to-scalar ratio of primordial fluctuations $r$. They can be written in terms of the slow-roll parameters:

$$
\begin{aligned}
A_{S} & =\frac{\kappa^{4} \mathcal{V}_{*}}{24 \pi^{2} \epsilon_{*}}, \\
n_{S} & =1+2 \eta_{*}-6 \epsilon_{*} \simeq 1+2 \eta_{*}, \\
r & =16 \epsilon_{*},
\end{aligned}
$$

evaluated using the field value $c_{*}$ at the horizon exit.

We can choose for example

$$
v=1.86945, x=0.08435, y=4.07, m=3.77 \times 10^{-8} .
$$

For this choice, we have $\Delta=-4.41049$.

The scalar potential for these parameters as a function of $c$ and $\rho$ is plotted in Fig. $2 \mathrm{a}$ and $2 \mathrm{~b}$, respectively. The relation between $c$ and $\rho$ coordinates is shown in Fig. 2c where the physical domain is $c<0$. The slow-roll parameters in $\rho$ coordinates are plotted in Fig. 2d.

Choosing the initial condition $\mathcal{c}_{*}=-0.00017$ and $c_{\text {end }}=-0.01192$ (or equivalently, by using (61), $\rho_{*}=0.0225$ and $\rho_{\text {end }}=$ 0.1869 ), we obtain $N=59.48, n_{s}=0.9597, r=4.15 \times 10^{-6}$ and $A_{s}=2.2 \times 10^{-9}$, which are within the $2 \sigma$-region of Planck'18 data [17].

Using the constraint (25), we obtain $q_{-} \approx 31.5413$. From (29), (30) and (31), we find that the mass ratios indeed satisfy the integrating out condition,

$$
\left.\frac{m_{A_{\mu}}^{2}}{\mathcal{V}_{\varphi_{+}^{*} \varphi_{+}}^{\mathrm{UV}}}\right|_{\mathrm{vac}} \approx 38.2253,\left.\frac{\mathcal{V}_{\varphi_{-}^{*} \varphi_{-}}^{\mathrm{UV}}}{\mathcal{V}_{\varphi_{+}^{*} \varphi_{+}}^{\mathrm{UV}}}\right|_{\mathrm{vac}} \approx 21.9463,\left.\frac{\mathcal{V}_{\varphi_{-}^{*} \varphi_{-}^{*}}^{\mathrm{UV}}}{\mathcal{V}_{\varphi_{+}^{*} \varphi_{+}}^{\mathrm{UV}}}\right|_{\mathrm{vac}} \approx 9.8853
$$




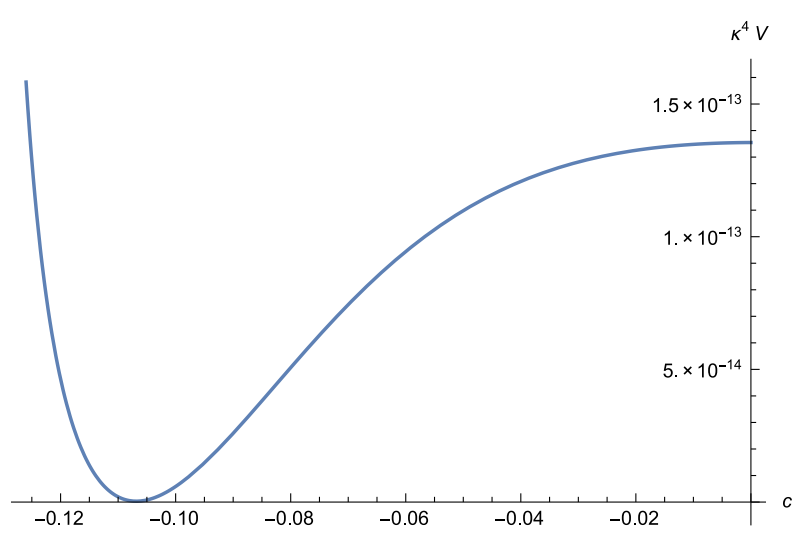

(a)

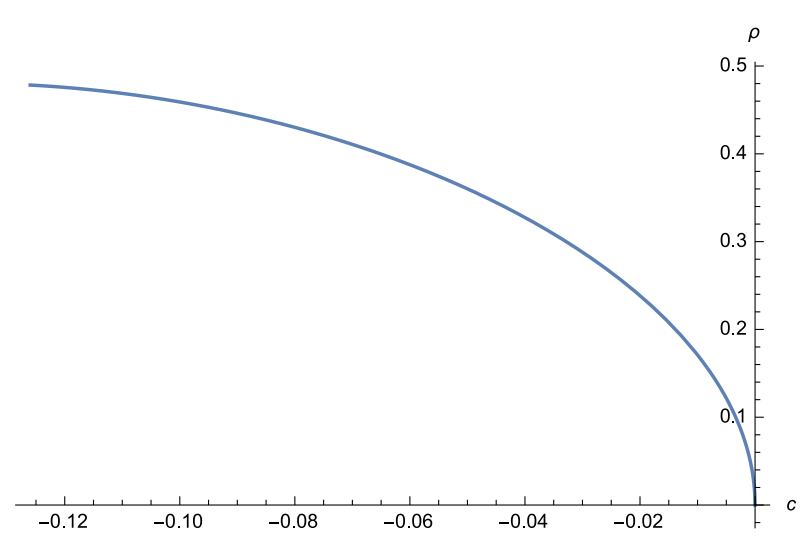

(c)

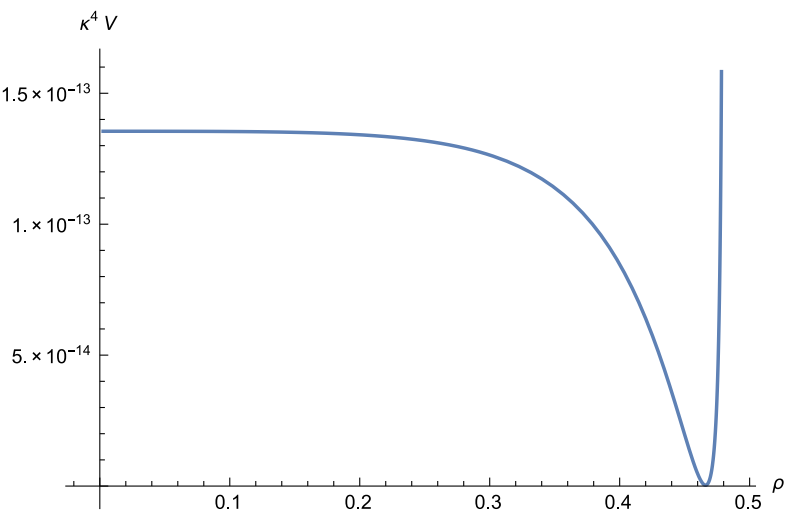

(b)

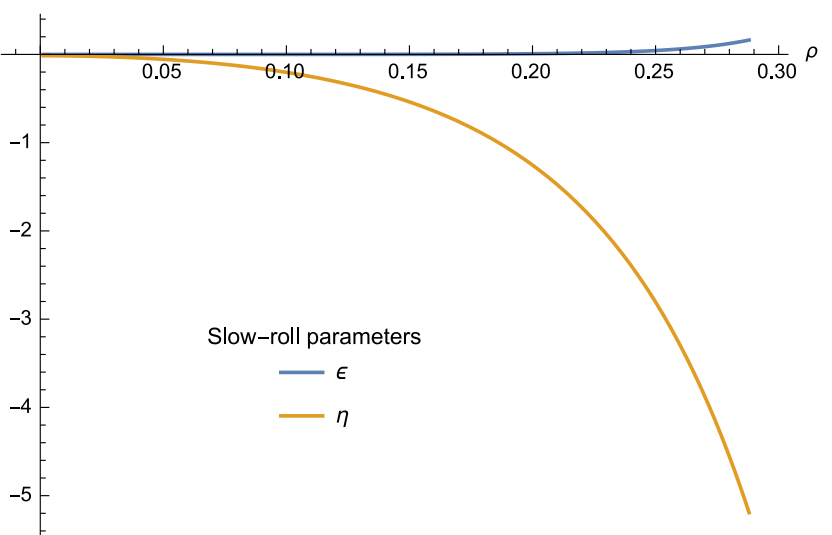

(d)

FIGURE 2: Plots of the scalar potential in Region III of Fig. 1 with parameters (74) as a function of the coordinate $c$ in (a) and $\rho$ in (b). The relation between $\rho$ and $c$ is plotted in (c). The slow-roll parameters $\epsilon$ and $\eta$ are shown in (d).

\section{FAYET-ILIOPOULOS (FI) D-TERMS IN SUPERGRAVITY}

In this section, we follow the notation in [14]. The chiral weight $w^{\prime}$ in this section is related to the chiral weight $\tilde{w}$ of the previous section by $\tilde{w}=\frac{2}{3} w^{\prime}$.

\subsection{Review}

In [8] (see also in [18]), a new (constant) FI term was proposed of the form $\mathcal{L}_{\mathrm{FI}}=\xi_{2} D+$ fermions, that can be coupled to supergravity without gauging the R-symmetry. It is non-singular when the $D$-auxiliary filed has a non vanishing vacuum expectation value (VEV), and the corresponding supergravity Lagrangian is:

$$
\mathcal{L}_{\mathrm{FI}}=\xi_{2}\left[S_{0} \bar{S}_{0} \frac{w^{2} \bar{w}^{2}}{\bar{T}\left(w^{2}\right) T\left(\bar{w}^{2}\right)}(V)_{D}\right]_{D}
$$

where $\xi_{2}$ is a constant parameter. In the superconformal formalism, the chiral compensator field $S_{0}$, with Weyl and chiral weights $\left(\delta, w^{\prime}\right)=(1,1)$, has components $S_{0}=\left(s_{0}, P_{L} \Omega_{0}, F_{0}\right)$. The vector multiplet has vanishing Weyl and chiral weights, and its components are given by $V=\left(v, \zeta, \mathcal{H}, v_{\mu}, \lambda, D\right)$. In the Wess-Zumino gauge, the first components are put to zero $v=\zeta=\mathcal{H}=0$. The multiplet $w^{2}$ is of weights $(1,1)$, and given by

$$
w^{2}=\frac{\bar{\lambda} P_{L} \lambda}{S_{0}^{2}}, \quad \bar{w}^{2}=\frac{\lambda P_{R} \bar{\lambda}}{\bar{S}_{0}^{2}} .
$$

The components of $\bar{\lambda} P_{L} \lambda$ are given by

$$
\bar{\lambda} P_{L} \lambda=\left(\bar{\lambda} P_{L} \lambda ; \sqrt{2} P_{L}\left(-\frac{1}{2} \gamma \cdot \hat{F}+i D\right) \lambda ; 2 \bar{\lambda} P_{L} \not D \lambda+\hat{F}^{-} \cdot \hat{F}^{-}-D^{2}\right)
$$


The kinetic terms for the gauge multiplet are given by

$$
\mathcal{L}_{\text {kin }}=-\frac{1}{4}\left[\bar{\lambda} P_{L} \lambda\right]_{F}+\text { h.c. } .
$$

The operator $T(\bar{T})$ is defined in $[19,20]$, and leads to a chiral (antichiral) multiplet. For example, the chiral multiplet $T\left(\bar{w}^{2}\right)$ has weights $(2,2)$. In global supersymmetry the operator $T$ corresponds to the usual chiral projection operator $\bar{D}^{2}$.

From now on, we will drop the notation of h.c. and implicitly assume its presence for every [ ] $F$ term in the Lagrangian. Finally, the multiplet $(V)_{D}$ is a linear multiplet with weights $(2,0)$, given by

$$
(V)_{D}=\left(D, \not D \lambda, 0, \mathcal{D}^{b} \hat{F}_{a b},-\not D D D \lambda,-\square^{C} D\right)
$$

The definitions of $\not D \lambda$ and the covariant field strength $\hat{F}_{a b}$ can be found in eq. (17.1) of [14], which reduce for an abelian gauge field to

$$
\begin{aligned}
\hat{F}_{a b} & =e_{a}^{\mu} e_{b}^{\nu}\left(2 \partial_{[\mu} A_{v]}+\bar{\psi}_{[\mu} \gamma_{\nu]} \lambda\right) \\
\mathcal{D}_{\mu} \lambda & =\left(\partial_{\mu}-\frac{3}{2} b_{\mu}+\frac{1}{4} w_{\mu}^{a b} \gamma_{a b}-\frac{3}{2} i \gamma_{*} \mathcal{A}_{\mu}\right) \lambda \\
& -\left(\frac{1}{4} \gamma^{a b} \hat{F}_{a b}+\frac{1}{2} i \gamma_{*} D\right) \psi_{\mu} .
\end{aligned}
$$

Here, $e_{a}^{\mu}$ is the vierbein, with frame indices $a, b$ and coordinate indices $\mu, v$. The fields $w_{\mu}^{a b}, b_{\mu}$, and $\mathcal{A}_{\mu}$ are the gauge fields corresponding to Lorentz transformations, dilatations, and $T_{R}$ symmetry of the conformal algebra respectively, while $\psi_{\mu}$ is the gravitino. The conformal d'Alembertian is given by $\square^{C}=\eta^{a b} \mathcal{D}_{a} \mathcal{D}_{b}$. is

Let us consider first the case of pure supergravity coupled to a $U(1)$ gauge multiplet with the FI term in (76). The full Lagrangian

$$
\mathcal{L}=-3\left[S_{0} \bar{S}_{0}\right]_{D}+\left[S_{0}^{3} W_{0}\right]_{F}-\frac{1}{4}\left[\bar{\lambda} P_{L} \lambda\right]_{F}+\mathcal{L}_{\mathrm{FI}}
$$

Supersymmetry is broken via a non-vanishing VEV of the D-auxiliary component of the vector multiplet driven by the linear term in $\mathrm{D}$, with the Goldstino being the $U(1)$ gaugino. After having gauge fixed the compensator through $S_{0}=1$, integrated the auxiliary fields, and in the unitary gauge where the Goldstino vanishes, the Lagarangian in component form reads [8]:

$$
\begin{aligned}
e^{-1} \mathcal{L}= & \frac{1}{2}\left(R-\bar{\psi}_{\mu} \gamma^{\mu \nu \rho} D_{\nu} \psi_{\rho}+m_{3 / 2} \bar{\psi}_{\mu} \gamma^{\mu v} \psi_{v}\right) \\
& -\frac{1}{4} F^{\mu \nu} F_{\mu \nu}-\left(-3 m_{3 / 2}^{2}+\frac{1}{2} \xi_{2}^{2}\right),
\end{aligned}
$$

with $m_{3 / 2}=W_{0}$, a constant superpotential. In the absence of matter, any $\xi_{2} \neq 0$ uplifts the vacuum energy by a constant term $\mathcal{V}_{F I}=\xi_{2}^{2} / 2$ and breaks supersymetry. It is important to note that the FI term given by eq. (76) does not require the gauging of an R-symmetry, but breaks invariance under Kähler transformations.

Let us now couple the term $\mathcal{L}_{\text {FI }}$ given by eq. (76) to additional matter fields charged under the $U(1)$. For simplicity, we focus on a single chiral multiplet $X$. The extension to more chiral multiplets is trivial. The Lagrangian is given by

$$
\mathcal{L}=-3\left[S_{0} \bar{S}_{0} e^{-\frac{1}{3} K(X, \bar{X})}\right]_{D}+\left[S_{0}^{3} W(X)\right]_{F}-\frac{1}{4}\left[f(X) \bar{\lambda} P_{L} \lambda\right]_{F}+\mathcal{L}_{\mathrm{FI}}
$$

with a Kähler potential $K(X, \bar{X})$, a superpotential $W(X)$ and a gauge kinetic function $f(X)$. The first three terms in eq. (84) give the usual supergravity Lagrangian [14]. We assume that the multiplet $X$ transforms under the $U(1)$,

$$
\begin{aligned}
& V \rightarrow V+i \Lambda-i \bar{\Lambda}, \\
& X \rightarrow X e^{-i q \Lambda},
\end{aligned}
$$

with gauge multiplet parameter $\Lambda$. We assume that the $U(1)$ is not an R-symmetry. In other words, we assume that the superpotential does not transform under the gauge symmetry. For a model with a single chiral multiplet this implies that the superpotential is constant

$$
W(X)=F
$$

\footnotetext{
${ }^{8}$ The operator $T$ indeed has the property that $T(Z)=0$ for a chiral multiplet $Z$. Moreover, for a vector multiplet $V$ we have $T(Z C)=Z T(C)$, and $[C]_{D}=\frac{1}{2}[T(C)]_{F}$.
} 
Gauge invariance fixes the Kähler potential to be a function of $X e^{q V} \bar{X}$ (for notational simplicity, in the following we omit the $e^{q V}$ factors).

Indeed, in this case the term $\mathcal{L}_{\mathrm{FI}}$ can be consistently added to the theory, similar to [8], and the resulting D-term contribution to the scalar potential acquires an extra term proportional to $\xi_{2}$

$$
\mathcal{V}_{D}=\frac{1}{2} \operatorname{Re}(f(X))^{-1}\left(i k_{X} \partial_{X} K+\xi_{2} e^{\frac{1}{3} K}\right)^{2}
$$

where the Killing vector is $k_{X}=-i q X$. The F-term contribution to the scalar potential remains the usual and for a constant superpotential (86), it reduces to

$$
\mathcal{V}_{F}=|F|^{2} e^{K(X, \bar{X})}\left(-3+g^{X \bar{X}} \partial_{X} K \partial_{\bar{X}} K\right)
$$

From eq. (87) it can be seen that if the Kähler potential includes a term proportional to $\xi_{1} \log (X \bar{X})$, the D-term contribution to the scalar potential acquires another constant contribution. For example, if

$$
K(X, \bar{X})=X \bar{X}+\xi_{1} \ln (X \bar{X})
$$

the D-term contribution to the scalar potential becomes

$$
\mathcal{V}_{D}=\frac{1}{2} \operatorname{Re}(f(X))^{-1}\left(q X \bar{X}+q \xi_{1}+\xi_{2} e^{\frac{1}{3} K}\right)^{2} .
$$

We will argue below that the contribution proportional to $\xi_{1}$ is the usual FI term in a non R-symmetric Kähler frame, which can be consistently added to the model including the new FI term proportional to $\xi_{2}$.

In the absence of the extra term, a Kähler transformation

$$
\begin{aligned}
K(X, \bar{X}) & \rightarrow K(X, \bar{X})+J(X)+\bar{J}(\bar{X}), \\
W(X) & \rightarrow W(X) e^{-J(X)},
\end{aligned}
$$

with $J(X)=-\xi_{1} \ln X$ allows one to recast the model in the form

$$
\begin{aligned}
K(X, \bar{X}) & =X \bar{X} \\
W(X) & =m_{3 / 2} X
\end{aligned}
$$

where $m_{3 / 2}=F$. The two models result in the same Lagrangian, at least classically ${ }^{9}$. However, in the Kähler frame of eqs. (92) the superpotential transforms nontrivially under the gauge symmetry. As a consequence, the gauge symmetry becomes an Rsymmetry.

Note that the extra term (76) violates the Kähler invariance of the theory, and the two models related by a Kähler transformation are no longer equivalent. The model written in the Kähler frame where the gauge symmetry becomes an R-symmetry in eqs. (92) can not be consistently coupled to $\mathcal{L}_{\mathrm{FI}}$. A generalized Kähler invariant FI term has been built in [21, 22].

\subsection{The scalar potential in a Non R-symmetry frame}

In this section, we work in the Kähler frame where the superpotential does not transform, and take into account the two types of FI terms which were discussed in the last section. For convenience, we repeat here the Kähler potential in eq. (89) and restore the inverse reduced Planck mass $\kappa=M_{\mathrm{Pl}}^{-1}=\left(2.4 \times 10^{18} \mathrm{GeV}\right)^{-1}$ :

$$
K=\kappa^{-2}\left(X \bar{X}+\xi_{1} \ln X \bar{X}\right) .
$$

The superpotential and the gauge kinetic function are set to be constant ${ }^{10}$ :

$$
W=\kappa^{-3} F, \quad f(X)=1 .
$$

After performing a change of the field variable $X=\rho e^{i \theta}$ where $\rho \geq 0$ and setting $\xi_{1}=b$, the full scalar potential $\mathcal{V}=\mathcal{V}_{F}+\mathcal{V}_{D}$ is a function of $\rho$. The F-term contribution to the scalar potential is given by

$$
\mathcal{V}_{F}=\frac{1}{\mathcal{K}^{4}} F^{2} e^{\rho^{2}} \rho^{2 b}\left[\frac{\left(b+\rho^{2}\right)^{2}}{\rho^{2}}-3\right],
$$

\footnotetext{
${ }^{9}$ At the quantum level, a Kähler transformation also introduces a change in the gauge kinetic function $f$, see for example [13].

${ }^{10}$ Strictly speaking, the gauge kinetic function gets a field-dependent correction proportional to $q^{2} \ln \rho$, in order to cancel the chiral anomalies [4]. However, the correction turns out to be very small and can be neglected below, since the charge $q$ is chosen to be of order of $10^{-5}$ or smaller.
} 


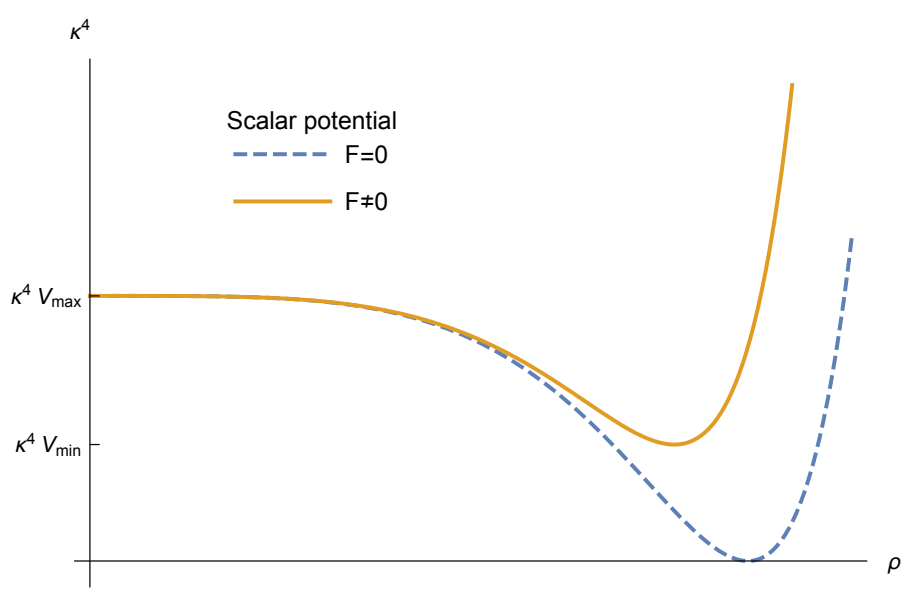

FIGURE 3: This plot shows the scalar potentials in $F=0$ and $F \neq 0$ cases. When $F=0$, we have a local maximum at $\rho_{\max }=0$ and a global minimum with zero cosmological constant. For $F \neq 0$, the origin $\rho=0$ is still the maximum but the global minimum now has a positive cosmological constant.

and the D-term contribution is

$$
\mathcal{V}_{D}=\frac{q^{2}}{2 \kappa^{4}}\left(b+\rho^{2}+\xi \rho^{\frac{2 b}{3}} e^{\frac{1}{3} \rho^{2}}\right)^{2}
$$

Note that we rescaled the second FI parameter by $\xi=\xi_{2} / q$. We consider the case with $\xi \neq 0$ because we are interested in the role of the new FI-term in inflationary models driven by supersymmetry breaking.

For $F=0$, one finds that for $\xi<-1$ and $b=3$ the potential has a maximum at $\rho=0$, and a supersymmetric minimum. Let us comment on supersymmetry (SUSY) breaking in the scalar potential. Since the superpotential is zero, the SUSY breaking is measured by the D-term order parameter, namely the Killing potential associated with the gauged U(1), which is defined by

$$
\mathcal{D}=i \kappa^{-2} \frac{-i q X}{W}\left(\frac{\partial W}{\partial X}+\kappa^{2} \frac{\partial \mathcal{K}}{\partial X} W\right)+\kappa^{-2} q \xi \rho^{2} e^{\rho^{2} / 3}
$$

This enters the scalar potential as $\mathcal{V}_{D}=\mathcal{D}^{2} / 2$. So, at the local maximum and during inflation $\mathcal{D}$ is of order $q$ and supersymmetry is broken. On the other hand, at the global minimum, supersymmetry is preserved and the potential vanishes. Strictly speaking, the supersymetric minimum is not valid because the new FI term becomes singular since the D-auxiliary vanishes. Therefore a small $F$ is required in any case.

For $F \neq 0$, the potential has still a local maximum at $\rho=0$ for $b=3$ and $\xi<-1$. For this choice, the derivatives of the potential have the following properties,

$$
\mathcal{V}^{\prime}(0)=0, \quad \mathcal{V}^{\prime \prime}(0)=6 \kappa^{-4} q^{2}(\xi+1)
$$

By our choice $\xi<-1$, the extremum is a local maximum, as desired.

Let us comment on the global minimum after turning on the F-term contribution. As long as we choose the parameters so that $F^{2} / q^{2} \ll 1$, the change in the global minimum $\rho_{v}$ is very small, of order $\mathcal{O}\left(F^{2} / q^{2}\right)$, because the extremisation condition depends only on the ratio $F^{2} / q^{2}$. So the change in the value of the global minimum is of order $\mathcal{O}\left(F^{2}\right)$. The plot of this change is given in Fig. 3.

In the present case $F \neq 0$, the order parameters of supersymetry breaking are both the Killing potential $\mathcal{D}$ and the F-term contribution $\mathcal{F}_{X}$, which read

$$
\mathcal{D} \propto q\left[3+\rho^{2}\left(1+\xi e^{\rho^{2} / 3}\right)\right], \quad \mathcal{F}_{X} \propto F \rho^{2}\left(3+\rho^{2}\right) e^{\rho^{2} / 2},
$$

where the F-term order parameter $\mathcal{F}_{X}$ is defined by

$$
\mathcal{F}_{X}=-\frac{1}{\sqrt{2}} e^{\kappa^{2} \mathcal{K} / 2}\left(\frac{\partial^{2} \mathcal{K}}{\partial X \partial \bar{X}}\right)^{-1 / 2}\left(\frac{\partial \bar{W}}{\partial \bar{X}}+\kappa^{2} \frac{\partial \mathcal{K}}{\partial \bar{X}} \bar{W}\right) .
$$

Therefore, near the local maximum, $\mathcal{F}_{X} / \mathcal{D} \sim \frac{F}{q} \rho^{2}$. On the other hand, at the global minimum, both $\mathcal{D}$ and $\mathcal{F}_{X}$ are of the same order i.e. $\mathcal{F}_{X} / \mathcal{D} \sim \frac{F}{q}$, assuming that $\rho$ at the minimum is of order $\mathcal{O}(1)$, which is true in our models below. This makes tuning of the vacuum energy between the F- and D-contribution in principle possible, along the lines of [4, 7].

Let us consider the case $b=0$ where only the new FI parameter $\xi$ contributes to the potential. In this case, the condition for the local maximum of the scalar potential at $\rho=0$ can be satisfied for $-3<\xi<0$. In the case where $F$ is set to zero, the scalar 
potential (96) has a minimum at $\rho_{\min }^{2}=3 \ln \left(-\frac{3}{\xi}\right)$. In order to have $\mathcal{V}_{\min }=0$, we can choose $\xi=-\frac{3}{e}$. However, we find that this choice of parameter $\xi$ does not allow slow-roll inflation near the maximum of the scalar potential. Similar to our previous models [4] it may be possible to achieve both the scalar potential satisfying slow-roll conditions and a small cosmological constant at the minimum by adding correction terms to the Kähler potential and turning on a parameter $F$. However, here we will focus on $b=3$ case where, as we will see shortly, less parameters are required to satisfy the observational constraints.

\subsection{Example for slow-roll D-term inflation}

In this section we focus on the case where $b=3$ and derive the condition that leads to slow-roll inflation scenarios, where the start of inflation (or, horizon crossing) is near the maximum of the potential at $\rho=0$. We also assume that the scalar potential is D-term dominated by choosing $F=0$, for which the model has only two parameters, namely $q$ and $\xi$. The parameter $q$ controls the overall scale of the potential and it will be fixed by the amplitude $A_{S}$ of the CMB data. The only free-parameter left over is $\xi$, which can be tuned to satisfy the slow-roll condition.

In order to calculate the slow-roll parameters, we need to work with the canonically normalised field $\chi$ defined by

$$
\frac{d \chi}{d \rho}=\sqrt{2 g_{X \bar{X}}}
$$

The slow-roll parameters are given in terms of the canonical field $\chi$ by

$$
\epsilon=\frac{1}{2 \kappa^{2}}\left(\frac{d V / d \chi}{V}\right)^{2}, \quad \eta=\frac{1}{\kappa^{2}} \frac{d^{2} V / d \chi^{2}}{V} .
$$

Since we assume inflation to start near $\rho=0$, the slow-roll parameters for small $\rho$ can be expanded as

$$
\begin{aligned}
& \epsilon=\frac{4}{9}(\xi+1)^{2} \rho^{2}+\mathcal{O}\left(\rho^{3}\right), \\
& \eta=\frac{2(1+\xi)}{3}+\mathcal{O}\left(\rho^{2}\right) .
\end{aligned}
$$

Note also that $\eta$ is negative when $\xi<-1$. We can therefore tune the parameter $\xi$ to avoid the $\eta$-problem. The observation is that at $\xi=-1$, the effective charge of $X$ vanishes and thus the $\rho$-dependence in the D-term contribution (96) becomes of quartic order.

Note that we obtain the same relation between $\epsilon$ and $\eta$ as in the model of inflation from supersymmetry breaking driven by an F-term from a linear superpotential and $b=1$ (see eq. (14)) [4]. Thus, there is a possibility to have flat plateau near the maximum that satisfies the slow-roll condition and at the same time a small cosmological constant at the minimum nearby.

The number of e-folds $N$ during inflation is determined by

$$
N=\kappa^{2} \int_{\chi_{*}}^{\chi_{\text {end }}} \frac{\mathcal{V}}{\partial_{\chi} \mathcal{V}} d \chi=\kappa^{2} \int_{\rho_{*}}^{\rho_{\text {end }}} \frac{\mathcal{V}}{\partial_{\rho} \mathcal{V}}\left(\frac{d \chi}{d \rho}\right)^{2} d \rho,
$$

where we choose $\left|\epsilon\left(\chi_{\text {end }}\right)\right|=1$. Notice that the slow-roll parameters for small $\rho^{2}$ satisfy the simple relation $\epsilon=\eta(0)^{2} \rho^{2}+O\left(\rho^{4}\right)$ by eq. (103). Therefore, the number of e-folds between $\rho=\rho_{1}$ and $\rho_{2}\left(\rho_{1}<\rho_{2}\right)$ takes the following simple approximate form as in [4],

$$
N \simeq \frac{1}{|\eta(0)|} \ln \left(\frac{\rho_{2}}{\rho_{1}}\right)=\frac{3}{2|\xi+1|} \ln \left(\frac{\rho_{2}}{\rho_{1}}\right) .
$$

as long as the expansions in (103) are valid in the region $\rho_{1} \leq \rho \leq \rho_{2}$. Here we also used the approximation $\eta(0) \simeq \eta_{*}$, which holds in this case.

We can compare the theoretical predictions of our model to the observational data via the power spectrum of scalar perturbations of the CMB, namely the amplitude $A_{s}$, tilt $n_{s}$ and the tensor-to-scalar ratio of primordial fluctuations $r$. From the relation of the spectral index above, one should have $\eta_{*} \simeq-0.02$, and thus eq. (105) gives approximately the desired number of e-folds when the logarithm is of order one. Actually, using this formula, we can estimate the upper bound of the tensor-to-scalar ratio $r$ and the Hubble scale $H_{*}$ following the same argument given in [4]; the upper bounds are given by computing the parameters $r, H_{*}$ assuming that the expansions (103) hold until the end of inflation. We then get the bound

$$
r \lesssim 16\left(\left|\eta_{*}\right| \rho_{\text {end }} e^{-\left|\eta_{*}\right| N}\right)^{2} \simeq 10^{-4}, \quad H_{*} \lesssim 10^{12} \mathrm{GeV},
$$

where we used $\left|\eta_{*}\right|=0.02, N \simeq 50-60$ and $\rho_{\text {end }} \lesssim 0.5$, which are consistent with our models.

\subsection{A small field inflation model from supergravity with observable tensor-to-scalar ratio}

Supergravity models with higher $r$ are of particular interest. In this section we show that our model can get large $r$ at the price of introducing some additional terms in the Kähler potential. Let us consider the previous model with additional quadratic and cubic terms in $X \bar{X}$ :

$$
K=\kappa^{-2}\left(X \bar{X}+A(X \bar{X})^{2}+B(X \bar{X})^{3}+b \ln X \bar{X}\right),
$$




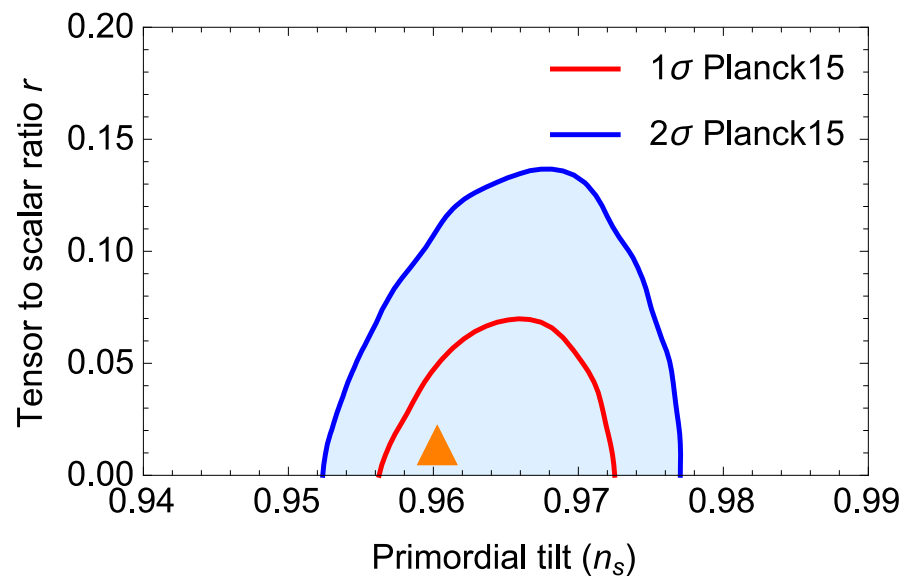

FIGURE 4: A plot of the predictions for the scalar potential with $F=0, b=3, A=0.176, B=0.091, \xi=-1.101$ and $q=8.68 \times 10^{-6}$ in the $n_{s}-r$ plane, versus Planck'15 results.

while the superpotential and the gauge kinetic function remain as in eq. (94). We now assume that inflation is driven by the D-term, setting the parameter $F=0$. In terms of the field variable $\rho$, we obtain the scalar potential:

$$
\mathcal{V}=q^{2}\left(b+\rho^{2}+2 A \rho^{4}+3 B \rho^{6}+\xi \rho^{\frac{2 b}{3}} e^{\frac{1}{3}\left(A \rho^{4}+B \rho^{6}+\rho^{2}\right)}\right)^{2} .
$$

We now have two more parameters $A$ and $B$. This does not affect the arguments of the choices of $b$ in the previous sections because these parameters appear in higher orders in $\rho$ in the scalar potential. So, we consider the case $b=3$. The simple formula (105) for the number of e-folds for small $\rho^{2}$ also holds even when $A, B$ are turned on because the new parameters appear at order $\rho^{4}$ and higher. To obtain $r \approx 0.01$, we can choose for example

$$
q=8.68 \times 10^{-6}, \xi=-1.101, A=0.176, B=0.091
$$

By choosing the initial condition $\rho_{*}=0.445$ and $\rho_{\text {end }}=1.155$, we obtain the results $N=58, n_{s}=0.96, r=0.01$ and $A_{s}=2.2 \times 10^{-9}$ , which agree with Planck'15 data as shown in Fig. 4.

Note that an application of the new FI term in no-scale supergravity model for inflation can be found for example in [22, 23].

\section{ACKNOWLEDGEMENTS}

This work was supported in part by the Labex "Institut Lagrange de Paris", in part by a CNRS PICS grant, and in part by the "CUniverse" research promotion project by Chulalongkorn University (grant reference CUAASC).

\section{References}

[1] D. H. Lyth and A. Riotto, "Particle physics models of inflation and the cosmological density perturbation," Phys. Rept. 314 (1999) 1; A. D. Linde, "Particle physics and inflationary cosmology," Contemp. Concepts Phys. 5 (1990) 1 [hep-th/0503203].

[2] A. A. Starobinsky,"A New Type of Isotropic Cosmological Models Without Singularity," Phys. Lett. 91B (1980) 99.

[3] L. Randall and S. D. Thomas, "Solving the cosmological moduli problem with weak scale inflation," Nucl. Phys. B 449 (1995) 229 [hepph/9407248]; A. Riotto, "Inflation and the nature of supersymmetry breaking," Nucl. Phys. B 515 (1998) 413 [hep-ph/9707330]; K. I. Izawa, "Supersymmetry - breaking models of inflation," Prog. Theor. Phys. 99 (1998) 157 [hep-ph/9708315]; W. Buchmuller, L. Covi and D. Delepine, "Inflation and supersymmetry breaking," Phys. Lett. B 491 (2000) 183 [hep-ph/0006168].

[4] I. Antoniadis, A. Chatrabhuti, H. Isono and R. Knoops, “Inflation from Supersymmetry Breaking," Eur. Phys. J. C 77 (2017) no.11, 724 [arXiv:1706.04133 [hep-th]].

[5] I. Antoniadis and R. Knoops, Nucl. Phys. B 886 (2014) 43 [arXiv:1403.1534 [hep-th]]; G. Villadoro and F. Zwirner, Phys. Rev. Lett. 95 (2005) 231602 [hep-th/0508167].

[6] F. Catino, G. Villadoro and F. Zwirner, JHEP 1201 (2012) 002 [arXiv:1110.2174 [hep-th]].

[7] I. Antoniadis, A. Chatrabhuti, H. Isono and R. Knoops, "Inflation from Supergravity with Gauged R-symmetry in de Sitter Vacuum," Eur. Phys. J. C 76 (2016) no.12, 680 [arXiv:1608.02121 [hep-ph]].

[8] N. Cribiori, F. Farakos, M. Tournoy and A. van Proeyen, "Fayet-Iliopoulos terms in supergravity without gauged R-symmetry," JHEP 1804 (2018) 032 [arXiv:1712.08601 [hep-th]]

[9] I. Antoniadis, A. Chatrabhuti, H. Isono and R. Knoops, “Fayet-Iliopoulos terms in supergravity and D-term inflation," Eur. Phys. J. C 78 (2018) no.5, 366 [arXiv:1803.03817 [hep-th]].

[10] Y. Aldabergenov and S. V. Ketov, "Removing instability of inflation in Polonyi-Starobinsky supergravity by adding FI term," Mod. Phys. Lett. A 91 (2018) no.05, 1850032 [arXiv:1711.06789 [hep-th]]. 
[11] J. Wess and J. Bagger, "Supersymmetry and supergravity," Princeton University Press (1992).

[12] I. Antoniadis, D. M. Ghilencea and R. Knoops, "Gauged R-symmetry and its anomalies in 4D N=1 supergravity and phenomenological implications," JHEP 1502 (2015) 166 [arXiv:1412.4807 [hep-th]].

[13] V. Kaplunovsky and J. Louis, "Field dependent gauge couplings in locally supersymmetric effective quantum field theories," Nucl. Phys. B 422 (1994) 57 [hep-th/9402005].

[14] D. Z. Freedman and A. Van Proeyen, "Supergravity," Cambridge Univ. Press (2012).

[15] I. Antoniadis, A. Chatrabhuti, H. Isono and R. Knoops, "A microscopic model for inflation from supersymmetry breaking," Eur. Phys. J. C 79 (2019) no.7, 624 [arXiv:1905.00706 [hep-th]].

[16] K. Benakli, Y. Chen and M. D. Goodsell, “Minimal constrained superfields and the Fayet-Iliopoulos model," Eur. Phys. J. C 78 (2018) no.9, 711 [arXiv:1711.08466 [hep-th]].

[17] Y. Akrami et al. [Planck Collaboration], "Planck 2018 results. X. Constraints on inflation," arXiv:1807.06211 [astro-ph.CO].

[18] S. M. Kuzenko, "Taking a vector supermultiplet apart: Alternative Fayet-Iliopoulos-type terms," Phys. Lett. B 781 (2018) 723 [arXiv:1801.04794 [hep-th]].

[19] T. Kugo and S. Uehara, "N=1 Superconformal Tensor Calculus: Multiplets With External Lorentz Indices and Spinor Derivative Operators," Prog. Theor. Phys. 73 (1985) 235.

[20] S. Ferrara, R. Kallosh, A. Van Proeyen and T. Wrase, "Linear Versus Non-linear Supersymmetry, in General," JHEP 1604 (2016) 065 [arXiv:1603.02653 [hep-th]].

[21] I. Antoniadis, A. Chatrabhuti, H. Isono and R. Knoops, "The cosmological constant in Supergravity," Eur. Phys. J. C 78 (2018) no.9, 718 [arXiv:1805.00852 [hep-th]].

[22] I. Antoniadis and F. Rondeau, "New Kähler invariant Fayet-Iliopoulos terms in supergravity and cosmological applications," arXiv:1912.08117 [hep-th].

[23] Y. Aldabergenov, A. Chatrabhuti and S. V. Ketov, "Generalized dilaton-axion models of inflation, de Sitter vacua and spontaneous SUSY breaking in supergravity," Eur. Phys. J. C 79 (2019) no.8, 713 [arXiv:1907.10373 [hep-th]]. 